\title{
MUON TRANSFER IN HYDROGEN ISOTOPIC MIXTURES
}

\author{
W. CZAPLIŃSKI \\ Faculty of Physics and Nuclear Techniques, University of Mining and Metallurgy \\ Al. Mickiewicza 30, 30-059 Kraków, Poland \\ A. Kravtsov, A. Mikhailov \\ Petersburg Nuclear Physics Institute, 188350 Gatchina, Russia \\ AND N. POPOV \\ Sektion Physik der Universität München \\ Schellingstr. 4, 80799 Münchè', Germany
}

(Received November 10, 1997)

\begin{abstract}
Direct and inverse muon transfer between atomic orbits of muonic hydrogen isotopes is considered in the WKB approximation. The corresponding cross sections, calculated as functions of collision energy, are different above the thresholds for the inverse transfer and approach common limits depending on the principal quantum number and hydrogen isotopes. The comparison of results obtained by integration over impact parameter and by summation over angular momentum is also presented.
\end{abstract}

PACS numbers: $34.60 .+z$

\section{Introduction}

The study of kinetics of muonic hydrogen in hydrogen isotopic mixtures is an important problem of muonic atom physics, being connected with the investigation of the weak interactions in muon capture by hydrogen isotopes [1]. Some other fundamental aspects of theoretical physics, e.g. charge invariance of strong interactions [2], and astrophysics [3] are involved here.

The main processes that determine the $\mu$-atomic cascade are the subject of the intensive experimental and theoretical investigation by the use of various methods of atomic physics. Certain criticism arises incidentally [4] concerning the methods of theoretical analysis, e.g. with respect to the Coulomb deexcitation process. 
Muon transfer belongs to one of the basic processes which determine the cascade of the excited muonic hydrogen atom. It has been analyzed in numerous papers since 1962 [5]. In the first paper concerning the muon exchange in excited states of muonic hydrogen [6] the Landau-Zener approximation [7] was used. A method of a complex internuclear distance $R$ [8] was used in Refs. [9-11] for description of $\mu$-atomic processes. An attempt to compare the $\mu$-atomic cascade calculations with experimental data was given in Ref. [12].

In this paper we present the direct and inverse transfer rates in the reactions

$$
(h \mu)_{n}+h^{\prime} \leftrightarrow\left(h^{\prime} \mu\right)_{n}+h,
$$

where $h, h^{\prime} \equiv p, d, t$ are nuclei of hydrogen isotopes ( $h^{\prime}$ being heavier than $h$ ) and $n$ is a principal quantum number, calculated by different quasiclassical methods.

\section{Methods of calculation}

Muon transfer (direct and inverse) in excited states of muonic hydrogen (1) was considered in the framework of WKB approximation in Refs. [10, 11]. However, the more accurate calculation of the inverse transfer (which was first calculated in Refs. [11]) near the threshold is needed. The process (1) is determined by a series of complex branch points $R_{c}$, located equidistantly in complex $R$-plane [8], that connect the initial and final molecular terms of the two center Coulomb problem. The muon is transferred to another nucleus at the internuclear distance close to $R_{c}$.

The cross section of the reaction (1) in the framework of WKB approximation is given by

$$
\sigma=\pi \int_{0}^{\rho_{\max }^{2}} P \mathrm{~d} \rho^{2},
$$

where $\rho$ is the impact parameter and $P$ is reaction probability $[8,10,13]$

$$
P=\left[2 \cosh ^{2} \delta(\rho)\right]^{-1}
$$

with the Massey parameter

$$
\delta=\left|\operatorname{Im} \int_{C} p \mathrm{~d} R\right|
$$

The contour $C$ goes round the branch point $R_{c}$, closest to the real axis; $p=\sqrt{2 \overline{M\left(\varepsilon-U-\varepsilon \rho^{2} / R^{2}\right)}}$ is the relative radial momentum of colliding atoms, where $\varepsilon$ is the collision energy at infinite $R$ and $M$ is the reduced mass: $M=M_{0} / m$, $M_{0}^{-1}=M_{h}^{-1}+M_{h^{\prime}}^{-1}, m^{-1}=m_{\mu}^{-1}+M_{\mathrm{in}}^{-1}$ with masses of muon and nuclei of hydrogen isotopes $m_{\mu}, M_{h}, M_{h^{\prime}}$, respectively. $M_{\text {in }}$ is the mass of the hydrogen isotope of muonic hydrogen in the input channel (we use the $\mu$-atomic units: $e=\hbar=m=1$ ). The potential $U$ (being a complex function of $R$ on different Riemann's surfaces of the complex $R$-plane) and the corresponding branch points, $R_{c}$ [8], have been calculated with inclusion of electron screening correction [6]. The collision energy, $\varepsilon$, is counted from the $R \rightarrow \infty$ limit of the potential in the input channel. The maximum impact parameter is determined from the requirement that the radial momentum should be real on the trajectory, i.e. for $R \geq \operatorname{Re} R_{c}$, where the transition takes place. Actually only the momentum corresponding to the left hand side of Eq. (1) is important for determination of $\rho_{\max }^{2}$ for direct and inverse reactions. 
It is clear however that integration over the impact parameter in Eq. (2) is justified when the number of partial waves included is high enough [14]. When it is not the case, one has to sum over partial waves using the quasiclassic phase shifts for elastic cross section or transition probabilities for inelastic reactions. Then the cross section is given by the following formula:

$$
\sigma=\frac{\pi}{k^{2}} \sum_{l=0}^{l_{\max }}(2 l+1) P_{l},
$$

where $k=\sqrt{2 M \varepsilon}$ and $l$ is the relative orbital momentum of muonic hydrogen and target atom (molecule). The reaction probability $P_{l}$ could be expressed by Eq. (3) with replacement of $\delta(\rho)$ by $\delta_{l}$. The Massey parameter $\delta_{l}$ is determined by Eq. (4) with the relative radial momentum $p=\sqrt{2 M(\varepsilon-U)-(l+1 / 2)^{2} / R^{2}}$. In order to obtain the reaction cross section for a given principal quantum number $n$, we sum cross sections (5) corresponding to all attracting molecular terms labeled by the same $n$. The $l_{\max }$ were determined in the analogous way as $\rho_{\max }$.

As follows from Ref. [15] the agreement of the calculated elastic cross sections obtained by summing over partial waves and by integration over $\rho$ is very good, especially for $\varepsilon>0.1 \mathrm{eV}$ and $n \leq 6$. In fact $l_{\max }$ for elastic scattering change from several for thermal energy to about 100 for large collision energy $(\varepsilon \approx 10 \mathrm{eV})$ [15]. On the other hand, $l_{\max }$ for direct muon transfer (considered for only $n \leq 5$ ) was found to be $\leq 15$ in Ref. [9]. Therefore, cross sections for processes (1) are calculated in this paper using Eq. (5) for comparison.

The screening of the nucleus charge by the atomic electron was shown to be very important for $\mu$-atomic processes at low energy [16]. The screening effect in the first order of perturbation theory was first calculated in Ref. [17]. The alternative approach to the electron screening was also presented in Refs. [18, 19]. It was shown that inclusion of electron screening in the elastic scattering of the muonic hydrogen in the ground state leads to the increase in the cross section by an order of magnitude for $\varepsilon \ll 1 \mathrm{eV}$ for the lowest partial waves. Therefore, ground state muonic hydrogen scatters mainly on electron shell instead of nucleus especially in non-resonant cases [16]. Electron screening in elastic scattering of excited muonic hydrogen must be taken into account because the leading term in the unscreened interaction potential is proportional to $1 / R^{2}$ and the corresponding cross section diverges.

On the other hand, the muon transfer is due to collision with a target nucleus and the inclusion of the electron screening in the muon transfer in excited states reduces the corresponding cross section. Namely, the electron screening leads to the barrier in the effective potential $U+\varepsilon \rho^{2} / R^{2}[6]$. As a result, the maximum impact parameter, and hence the reaction cross section decreases, especially at low energies.

For $\varepsilon>1 \mathrm{eV}$ the influence of the screening gradually decreases with increasing energy. The screening correction was calculated in the first order of perturbation theory [6]. One should note however that for high $n \approx 10$ such a screening correction may be not accurate enough, because the muonic atom dimension becomes comparable with the atomic one and terms corresponding to the higher order of the perturbation theory should be taken into account together with the 
molecular structure of the target. At the same time, as our results show, the inverse transfer compensates entirely the direct one in the initial stage of the $\mu$-atomic cascade, where the collision energy is close to $c a .1 \mathrm{eV}$, the energy of the nascent muonic atom. For this reason the screening effects seem to be not very important for large $n$. Precise determination of $\rho_{\max }$ or $l_{\max }$ is especially important for calculation of cross sections and reaction rates, $\lambda$, in the energy region near threshold of the inverse transfer. Reaction rates for inverse transfer obtained in Ref. [11] are valid for energies exceeding the corresponding thresholds. The $n$-dependence of inverse transfer rates presented in Fig. 4 of Ref. [11] is correct with the exception of the $p d$ case at $\varepsilon=2 \mathrm{eV}$ and $n=8$, where the corresponding transition rates were overestimated due to incorrect determination of $\rho_{\max }$.

\section{Results and discussion}

Reaction rates for the inverse muon transfer at the vicinity of the corresponding thresholds obtained by both methods described above are presented in Figs. 1 and 2 of the present paper for the first time.

Direct transfer rates also shown in Fig. 1 coincide with the corresponding curves in Fig. 2 of Ref. [10]. The results for $n>8$ differs, however, from the ones presented in Fig. 3 of Ref. [11] due to the computer program error in Ref. [11].

As seen from Figs. 1 and 2 the inverse transfer compensates the direct one for $\varepsilon>\varepsilon_{\mathrm{t}}$, where $\varepsilon_{\mathrm{t}}$ is the threshold for the inverse reaction determined by the resonance defect $\Delta U=\left(\mu_{2}-\mu_{1}\right) /\left(2 n^{2}\right)=48 n^{-2} \mathrm{eV}$ for $d t, 135 n^{-2} \mathrm{eV}$ for $p d$, and $183 n^{-2} \mathrm{eV}$ for $p t$ ( $\mu_{1}$ and $\mu_{2}$ are reduced masses of the light and heavy muonic atoms, respectively). The larger is $n$ the smaller is $\varepsilon_{t}$, so the total compensation could occur only in the initial stage of a muonic atom cascade, i.e. for large $n$. However, to obtain a reliable conclusion about the influence of the inverse muon transfer on the kinetics of muonic hydrogen in a certain isotopic mixture, e.g. a Monte-Carlo simulation is needed.

\section{TABLE}

Average values of $l_{\max }$ for various $n$, energies and isotopic mixtures. Averaging was performed over attracting terms corresponding to the same $n$ [11].

\begin{tabular}{c|c|c|c|c|c|c|c|c|c}
\hline \hline \multirow{2}{*}{$n$} & \multicolumn{3}{|c|}{$\varepsilon=0.04 \mathrm{eV}$} & \multicolumn{3}{c|}{$\varepsilon=1 \mathrm{eV}$} & \multicolumn{3}{c}{$\varepsilon=10 \mathrm{eV}$} \\
\cline { 2 - 10 } & $d t$ & $p d$ & $p t$ & $d t$ & $p d$ & $p t$ & $d t$ & $p d$ & $p t$ \\
\hline 2 & 4 & 3 & 3 & 7 & 5 & 5 & 8 & 6 & 6 \\
3 & 4 & 3 & 3 & 11 & 8 & 8 & 14 & 10 & 11 \\
5 & 8 & 6 & 6 & 17 & 13 & 14 & 26 & 19 & 20 \\
10 & 9 & 7 & 7 & 27 & 20 & 22 & 75 & 56 & 58 \\
12 & 10 & 8 & 8 & 34 & 25 & 27 & 103 & 78 & 81
\end{tabular}

Reaction rates for the inverse muon transfer obtained by two methods are very close, especially for large $\varepsilon$ and $n$, where $l_{\max }$ is large (see Table). For the lowest energy and $n$, where $l_{\max }$ is small, the difference is also small. The non-monotonicity of the direct transfer rates in $H-D$ and $H-T$ mixtures for 

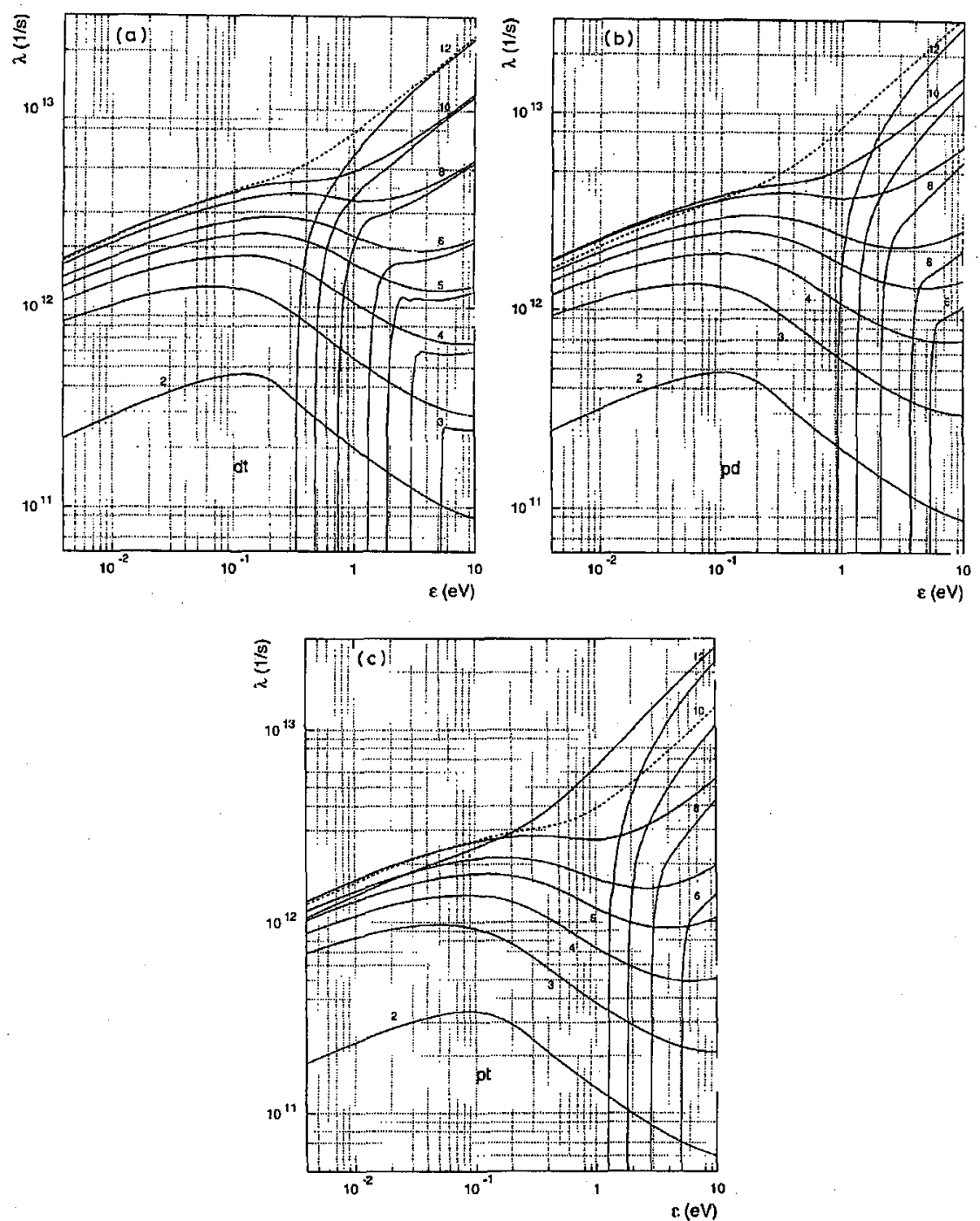

Fig. 1. Reaction rates $\lambda=\sigma N v$ (where $N=4.25 \times 10^{22} \mathrm{~cm}^{-3}$ is density of liquid hydrogen and $v=\sqrt{2 \varepsilon / M}$ is the relative velocity of the colliding atoms) for direct and inverse muon transfer from deuterium to tritium (a), protium to deuterium (b), and protium to tritium (c) as functions of collision energy $\varepsilon$ for different $n$ indicated on the curves. The results have been obtained using Eq. (2).

$n=2$ in the region $\varepsilon<0.02 \mathrm{eV}$ (see Fig. 2) is explained by increasing influence of $l=1$ partial wave with increasing collision energy.

The results for the direct muon transfer presented in this paper do not confirm the statement of Ref. [9] about $l_{\max } \leq 15$ for $n \leq 5$. Furthermore, our reaction rates for $(d \mu)_{n}+t \rightarrow(t \mu)_{n}+d$ transfer are about 1.5 times greater than those 

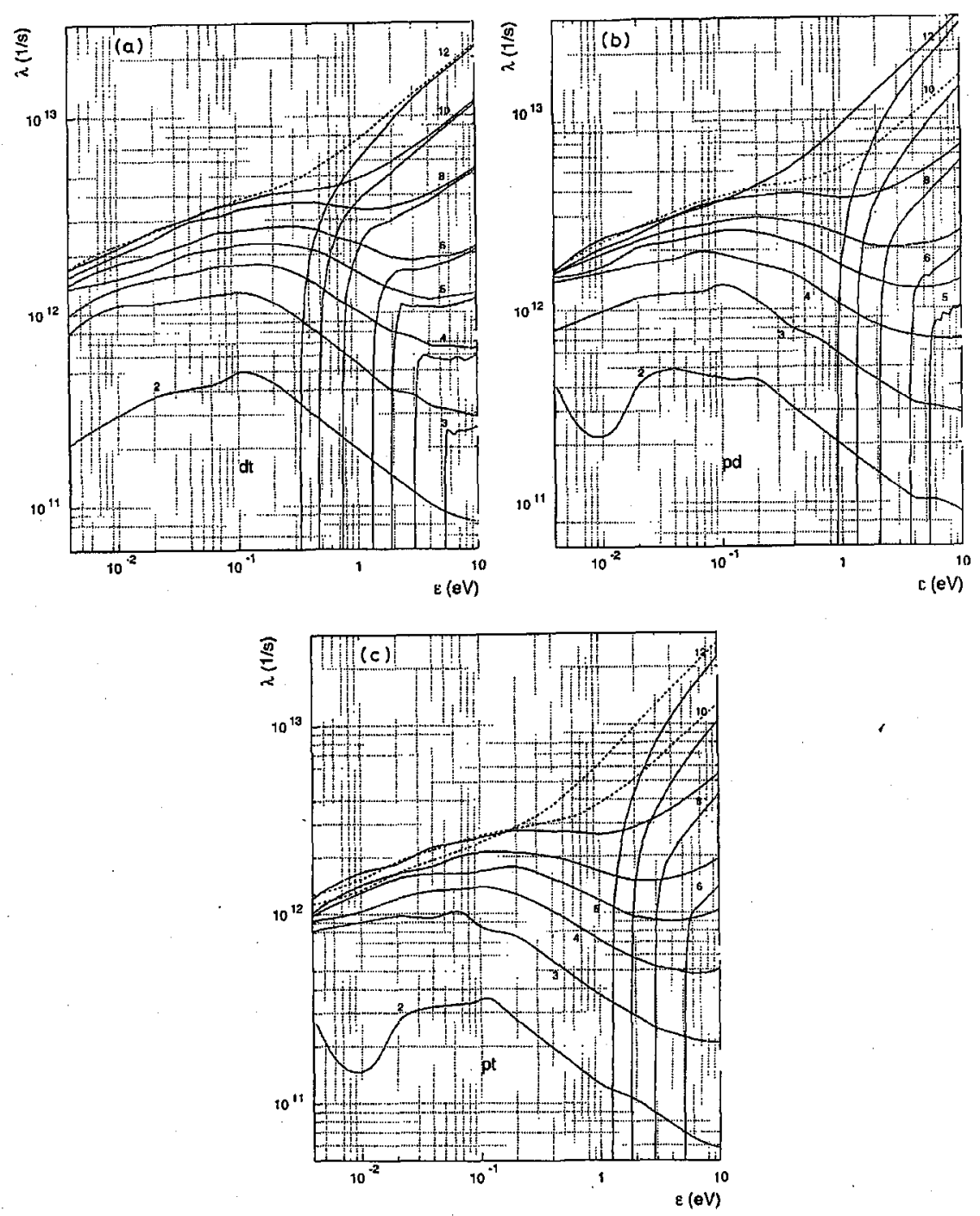

Fig. 2. Reaction rates $\lambda$ for direct and inverse muon transfer from deuterium to tritium (a), protium to deuterium (b), and protium to tritium (c) as functions of $\varepsilon$ for different $n$ indicated on the curves. The results have been obtained using Eq. (5).

of Ref. [9] for the low collision energies $(\varepsilon \leq 0.01 \mathrm{eV})$ although they practically coincide in the energy range near the maximum (with exception $n=2$, where they are greater by about $50 \%$ ). Thus it is clear that the energy dependence of the cross sections presented in this paper and in Ref. [9] are different. Any explanation of this disagreement cannot be given here because details of calculation are not presented in Ref. [9]. 


\section{Acknowledgments}

We are grateful to Dr. W. von Hörsten for supporting this work. Financial support from the Volkswagen-Stiftung (grant No. 1/70731) and the Committee for Scientific Research (Poland) are gratefully acknowledged.

\section{References}

[1] A. Bertin, A. Vitale, in: Fifty Years of Weak-Interaction Physics, Eds. A. Bertin, R.A. Ricci, A. Vitale, Italian Physical Society, Bologna 1984, p. 130.

[2] V.B. Belyaev, M. Decker, H. Fiedeldey, S.A. Rakityansky, W. Sandhas, S.A. Sofianos, Nukleonika 40, 3 (1995).

[3] C. Rolfs, in: Nuclear Astrophysics, in series Research Reports in Physics, Springer, Berlin 1988, p. 162.

[4] L.I. Ponomarev, E.A. Solov'ev, Pis'ma Zh. Eksp. Teor. Fiz. 64, 129 (1996) [JETP Lett. 64, 135 (1996)].

[5] S.S. Gershtein, Zh. Eksp. Teor. Fiz. 43, 706 (1962) [Sov. Phys. JETP 16, 501 (1963)].

[6] L.I. Menshikov, L.I. Ponomarev, Z. Phys. D 2, 1 (1986).

[7] H. Nakamura, C. Zhu, Comments At. Mol. Phys. 32, 249 (1996).

[8] E.A. Solov'ev, Usp. Fiz. Nauk 157, 437 (1989) [Sov. Phys. Usp. 32, 228 (1989)].

[9] V.V. Gusev, L.I. Ponomarev, E.A. Solov'ev, Hyperfine Interactions 82, 53 (1993).

[10] W. Czapliński, A. Guła, A. Kravtsov, A. Mikhailov, N. Popov, Phys. Rev. A 50, 518 (1994).

[11] W. Czapliński, A. Guła, A. Kravtsov, A. Mikhailov, N. Popov, Phys. Rev. A 50, 525 (1994).

[12] V.E. Markushin, Phys. Rev. A 50, 1137 (1994); E.C. Aschenauer, V.E. Markushin, Z. Phys. D 39, 165 (1997).

[13] Yu.N. Demkov, Zh. Eksp. Teor. Fiz. 45, 195 (1963) [Sov. Phys. JETP 18, 138 (1964)].

[14] L.D. Landau, E.M. Lifshitz, Quantum Mechanics, Pergamon, Oxford 1977.

[15] V. Bystritsky, W. Czapliński, J. Woźniak, E. Guła, A. Kravtsov, A. Mikhailov, N. Popov, Phys. Rev. A 53, 4169 (1996).

[16] A.V. Kravtsov, A.I. Mikhailov, N.P. Popov, J. Phys. B, At. Mol. Phys. 19, 1323 (1986); Phys. Lett. A 116, 180 (1986); N.P. Popov, Muon Cat. Fusion 2, 207 (1988).

[17] S. Cohen, D.L. Judd, R.J. Riddell, Phys. Rev. 119, 397 (1960).

[18] A. Adamczak, V.S. Melezhik, L.I. Menshikov, Z. Phys. D 4, 153 (1986); A. Adamczak, V.S. Melezhik, Muon Cat. Fusion 2, 131 (1988).

[19] J.S. Cohen, Muon Cat. Fusion 5/6, 3 (1990/91).

[20] M. Filipowicz, W. Czapliński, E. Guła, A. Kravtsov, A. Mikhailov, N. Popov, accepted for publication in Nuovo Cimento D. 